\title{
Positive correlation of airway resistance and serum asymmetric dimethylarginine (ADMA) in bronchial asthma patients lacking evidence for systemic inflammation
}

\author{
Gabor Tajti ${ }^{1}$, Csaba Papp ${ }^{1}$, Laszlo Kardos², Sandor Keki ${ }^{3}$, Krisztian Pak ${ }^{4}$, Magdolna Emma Szilasi ${ }^{4}$, \\ Rudolf Gesztelyi ${ }^{4}$, Angela Mikaczo ${ }^{5}$, Andrea Fodor ${ }^{5}$, Maria Szilasi ${ }^{5}$ and Judit Zsuga ${ }^{*}$
}

\begin{abstract}
Background: Contribution of nitric-oxide (NO) pathway to the pathogenesis of bronchial asthma (asthma) is ambiguous as NO may confer both protective and detrimental effects depending on the NO synthase (NOS) isoforms, tissue compartments and underlying pathological conditions (e.g. systemic inflammation). Asymmetric dimethylarginine (ADMA) is an endogenous inhibitor and uncoupler of NOS with distinct selectivity for NOS isoforms. In a cross-sectional study, we assessed whether ADMA is an independent predictor of airway resistance $\left(R_{a w}\right)$ in therapy-controlled asthma.

Methods: 154 therapy-controlled asthma patients were recruited. ADMA, symmetric dimethylarginine and arginine were quantitated by HPLC with fluorescent detection. Pulmonary function test was done using whole-body plethysmography, quality of life via St. George's Respiratory questionnaire (SGRQ). Multiple linear regression was used to identify independent determinants of $R_{a w}$. The final model was stratified based on therapy control.

Results: Evidence for systemic inflammation indicated by CRP and procalcitonin was lacking in our sample. Log $\mathrm{R}_{\text {aw }}$ showed significant positive correlation with log ADMA in the whole data set and well-controlled but not in the not well-controlled stratum (Spearman correlation coefficients: $0.27, p<0.001 ; 0.30, p<0.001 ; 0.12, p=0.51$ respectively). This relationship remained significant after adjusting for confounders by multiple linear regression $(\beta=0.22, \mathrm{Cl} 0.054$, $0.383 p=0.01$ ). FEF $25-75 \% \%$ predicted and SGRQ Total score showed significant negative while SGRQ Activity score showed significant positive correlation with $\mathrm{R}_{\mathrm{aw}}$ in the final model.
\end{abstract}

Conclusions: Positive correlation between $\mathrm{R}_{\mathrm{aw}}$ and $\mathrm{ADMA}$ in the absence of systemic inflammation implies that higher ADMA has detrimental effect on NO homeostasis and can contribute to a poor outcome in asthma.

Keywords: ADMA, Airway resistance, Bronchial asthma, SGRQ, Whole-body plethysmography

\section{Background}

Bronchial asthma (hereinafter referred to as asthma) has high socioeconomic impact stemming from premature morbidity, poor quality of life, significant healthcare

\footnotetext{
*Correspondence: zsuga.judit@med.unideb.hu

${ }^{1}$ Department of Health Systems Management and Quality Management

for Health Care, Faculty of Public Health, University of Debrecen,

Nagyerdei krt. 98, Debrecen 4032, Hungary

Full list of author information is available at the end of the article
}

utilization and loss of work productivity $[1,2]$. The key pathomechanistic properties of asthma are the presence of chronic inflammation in the lower respiratory tract and consequent airflow limitation manifested as dyspnea (predominantly in the form of recurrent bouts) [3, 4]. Contribution of the nitric oxide (NO) pathway to the evolution of inflammation in asthma has long been proposed, nonetheless the net effect of altered NO homeostasis in the inflammatory state characteristic of asthma is yet to be elucidated. 
The source of controversy is that, similar to other tissues like vasculature $[5,6]$, NO may confer both protective and detrimental effects that depends on the activity of different NO synthase (NOS) isoforms, on the affected tissue compartment and on some underlying conditions [7]. Of the three NOS isoforms (each expressed in the lung), endothelial NOS (eNOS) and neuronal NOS (nNOS) are Ca-calmodulin dependent constitutive enzymes that liberate low (ranging from femto- to picomolar) concentrations of $\mathrm{NO}$ within seconds of receptor activation. The eNOS, apart from vasculature, is chiefly localized in the bronchial epithelium and type II alveolar cells [8], and NO released by this isoform leads to broncho- and vasodilation. The nNOS is chiefly located in peripheral nerves innervating bronchial smooth muscle and submucosal secretory glands. Density of innervation decreases from trachea to smaller bronchi conferring reduced NO-mediated neural bronchodilation in smaller airways [9]. The third isoform is the inducible NOS (iNOS) that, albeit continuously expressed in lung epithelial cells [10], is chiefly present upon its induction by pro-inflammatory cytokines. These latter molecules activate the nuclear transcription factor NF- $\mathrm{kB}$ that leads to iNOS expression and thereby sustained high release of NO (in nanomolar concentration) over the course of hours to days [8]. Based on the results of preclinical and clinical studies it seems that iNOS may be produced by alveolar type II epithelial cells, lung fibroblasts, airway and vascular smooth muscle cells, endothelial cells, mast cells and neutrophils $[8,11]$, and its expression is inhibited by glucocorticoids [12]. Under conditions of inflammation, NO, synthetized by iNOS, and superoxide anion, formed by activated macrophages and neutrophils, react to form peroxynitrite and subsequent inflammatory cell recruitment, airway constriction and remodeling [13]. Change in the level of all three isoforms in distinct compartments has been described in asthma [7]. In asthma patients, increased expression of iNOS was described in airway epithelium $[7,14,15]$, while decline in activity of constitutive isoforms was also observed [16]. Furthermore, L-arginine or tetrahydrobiopterin depletion may cause uncoupling of all three NOS isoforms, switching the enzyme's function to produce superoxide instead of NO $[11,17,18]$.

Asymmetric dimethylarginine (ADMA), a metabolite of protein turnover throughout the body, is considered as a significant factor in NO homeostasis that may interfere with several processes related to the evolution of inflammatory airway diseases. ADMA is viewed as an endogenous competitive inhibitor of NOS showing higher selectivity for the constitutive isoforms [11]. In addition, animal studies showed that ADMA is a natural uncoupler of all the three NOS isoforms leading to increased superoxide formation and oxidative as well as nitrosative stress [19]. ADMA may also compete with $\mathrm{L}$-arginine for intracellular transport, thus it may limit L-arginine's availability as a substrate for NOS [20] and thereby contribute to intracellular L-arginine depletion. Furthermore, exogenous ADMA per se was shown to cause airway hyperresponsiveness, to increase collagen formation and to induce reversible fibrosis in mice [19]. This latter effect may be due to ADMA's ability to enhance the activity of arginase [20]. While lung (more specifically: airway epithelium) has been shown to be a major source of ADMA [11, 20], conflicting reports have emerged related to the systemic ADMA concentration in asthma [21] with reports describing both higher [22] and normal/low-normal [23] serum levels (Fig. 1).

Starting from the above-mentioned findings, we set out to elucidate whether ADMA is a risk or protective factor in the inflammatory state characteristic of asthma, by assessing the relationship of ADMA with lung function parameters descriptive of airflow limitation.

\section{Methods \\ Study design and protocol}

The study was prepared in line with the STROBE statement for cross-sectional studies [24]. Every patient was invited to participate in our study who visited the outpatient unit of the Department of Pulmonology (University of Debrecen) between September 12012 and October 152013 for the management of chronic airway inflammatory diseases including asthma, chronic obstructive pulmonary disease (COPD), asthma-COPD overlap syndrome (ACOS) and allergic rhinitis (AR). Exclusion criteria were every acute inflammatory disease over the preceding 1 month and malignancies or benign tumors in the history. Overall 319 patients were recruited (asthma: $\mathrm{n}=167$, COPD: $\mathrm{n}=74$, ACOS: $\mathrm{n}=21$ and AR: $\mathrm{n}=57$ ). Data of patients with asthma were included in the current analysis.

For the present study, those patients who were already diagnosed with asthma at the time of recruitment and underwent whole-body plethysmography during the recruitment period were involved $(n=154)$. Accordingly, all these patients participated in a control-based asthma management program complying with GINA as well as the relevant Hungarian practice guidelines $[25,26]$. Thus, treatment at the time of inclusion was provided as clinically warranted. Defined daily dose of inhaled corticosteroids was determined to allow comparisons across treatment regimens [27]. $R_{a w}$ is reflective of changes in alveolar pressure over changes in flow as it is highly dependent on state of airways, thus it is an appropriate parameter for quantifying airflow limitation [28]. (The most important parameters determining the state of 


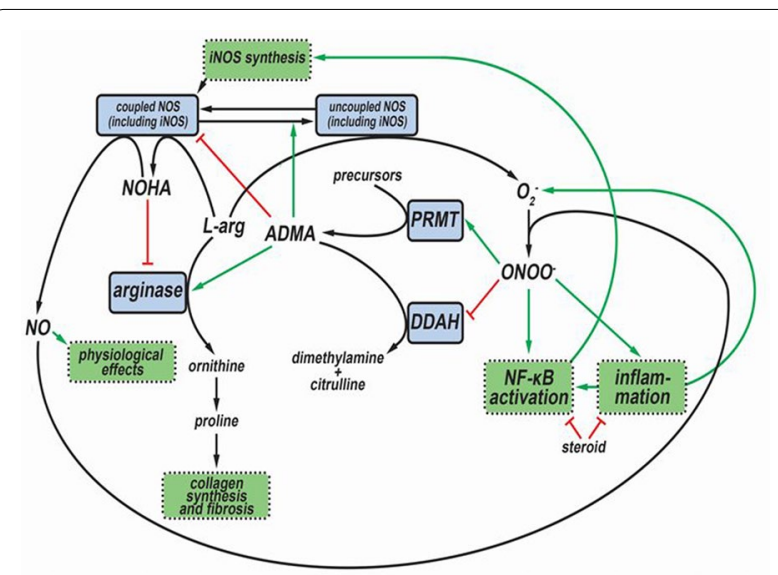

Fig. 1 Intertwining of asymmetric dimethylarginine (ADMA) with the nitric-oxide (NO) homeostasis in inflammatory airway diseases. Nitric oxide (NO) is formed from L-arginine through $N$-hydroxyarginine (NOHA) by NO-synthase enzymes (NOS) including the inducible one (iNOS). In bronchial asthma, nuclear transcription factor NF-kB, activated by inflammatory cytokines, increases the expression of iNOS. This augmented expression promotes formation of $\mathrm{NO}$, which compound, by reacting with superoxide anion $\left(\mathrm{O}_{2}{ }^{-}\right)$common under inflammatory conditions, provides peroxynitrite anion $\left(\mathrm{ONOO}^{-}\right)$and eventually results in nitrosative stress. This stress increases the production of asymmetric dimethylarginine (ADMA) by enhancing the expression of protein methyltransferase enzymes (PRMT), responsible for ADMA formation, and by decreasing the expression of dimethylargininase (DDAH), which participates in the elimination of ADMA. ADMA is an endogenous inhibitor and, in addition, a natural uncoupler of all NOS isoforms. Uncoupling of NOS leads to the production of $\mathrm{O}_{2}{ }^{-}$instead of $\mathrm{NO}$. Thus, elevated ADMA level, on one hand, decreases NO production, and, on the other hand, increases levels of oxidative and nitrosative agents via uncoupling NOS. Higher ADMA level also results in enhanced arginase activity, thereby contributing to collagen synthesis and (possibly) to the evolution of a (reversible) lung fibrosis. Corticosteroid therapy can prevent the iNOS-related processes elicited by inflammation (via inhibiting inflammatory processes and directly inhibiting the NF-KB expression) that leads to decreased iNOS expression and consequently lower production of NO, ADMA and oxidative/nitrosative agents. Green arrows: activation, enhancement; red lines: inhibition; black arrows: transformation, metabolic connection

airways are length and (average) diameter. If we want to characterize the airways with extensive (additive) properties only, these are: length, surface and volume [28]). Demographic and anthropometric data were acquired and body mass index was also calculated. Furthermore, history of smoking, diabetes, dyslipidemia and hypertension were also obtained. Cumulative measure of smoking exposure was characterized with pack-years (computed in a way that both past smoking and current smoking exposure were accounted for). Disease-specific quality of life was also assessed with the use of the official Hungarian version of St. George's Respiratory questionnaire (SGRQ) [29] after obtaining written permission from its proprietor (permission issued by Paul Jones, University of London, London, UK, on August 28 2012).

\section{Pulmonary function testing}

Pulmonary function was characterized using whole-body plethysmography. This enabled the assessment of residual volume and related measures such as functional reserve capacity and $\mathrm{R}_{\mathrm{aw}}$. Whole-body plethysmography was performed according to the ATS/ERS criteria [30-32] using Piston whole-body plethysmograph (PDT-111/p, Piston Medical, Hungary) equipped with automatic BTPS correction for cabin temperature, humidity and pressure as well as full automatic calibration and leakage test. Patients were instructed to take their medication as usual even on the morning of their examination (so plethysmography was performed while patients were on asthma control therapy). Best of three technically sound maneuvers was selected for each participant. For resistance curves, at least two separate and technically appropriate measurements were performed (each measurement consists recordings of at least 5 resistance loops) and results were accepted only if they were the same for both measurements. Pulmonary function data as well as percent predicted of normal reference values were obtained using algorithms supplied by the manufacturer. The following parameters were included in the statistical analysis: $\mathrm{R}_{\mathrm{aw}}(\mathrm{kPa} \mathrm{s} / \mathrm{L}), \mathrm{G}_{\mathrm{aw}}\left(=1 / \mathrm{R}_{\mathrm{aw}}\right)$, FEV $1 \%$ pred, FVC $\%$ pred, FEV1/FVC, FEF25-75\% \% pred, RV\% pred, TLC\% pred, RV/TLC \% pred, IC/TLC, IVC\% pred, FEV1/IVC\% pred, TGV\% pred, PEF\% pred and MEF50\% \% pred. Every patient underwent pulmonary function testing $(\mathrm{n}=154)$.

\section{Blood samples}

Blood samples were drawn in the morning of the examination after an overnight fast. Routine laboratory investigations were performed in line with the standard clinical practice at the Department of Laboratory Medicine (University of Debrecen). Accordingly, serum or plasma samples were used to determine parameters descriptive of carbohydrate (glucose, insulin, hemoglobin A1c (Hga1c)) and lipid homeostasis (total cholesterol, triglyceride, LDL-cholesterol, HDL-cholesterol, Lp(a), apoA1, apoB), of function of kidney (GFR, urea, creatinine), liver (GOT, GPT, $\gamma \mathrm{GT}$ ) and muscles (CK, LDH), and of inflammation (C-reactive protein (CRP), procalcitonin, fibrinogen). CRP was dichotomized as high vs. normal using the cutoff of 4.6 and $5.2 \mathrm{mg} / \mathrm{L}$ for female and male patients, respectively. HOMA index was calculated as described previously [6]. Other parameters related to L-arginine homeostasis were also assessed (folic acid, vitamin B12, urea). Serum samples used for the determination of $\mathrm{L}$-arginine, ADMA and symmetrical dimethylarginine (SDMA) were frozen within $60 \mathrm{~min}$ and stored at $-80^{\circ} \mathrm{C}$ until further analysis. 


\section{Determination of arginine, ADMA and SDMA}

Arginine and its dimethylated derivatives were measured as previously $[5,6]$. Briefly: serum samples $(250 \mu \mathrm{L})$ were mixed with $50 \mu \mathrm{L}$ L-homoarginine hydrochloride (Sigma, Steinheim, Germany) as internal standard $(1000 \mu \mathrm{mol} / \mathrm{L})$ and $700 \mu \mathrm{L}$ borate buffer ( $\mathrm{pH} 9.00)$, then solutions were passed through the solid-phase extraction cartridges (OASIS MCX $3 \mathrm{cc}$ ) using a 12-column manifold (J. T. Baker, Philipsburg, NJ). After washing, arginine derivatives were eluted with $1 \mathrm{~mL}$ ammonia-water-methanol solution (10/40/50, v/v/v) (Reanal, Budapest, Hungary; Scharlau, Sentmenat, Spain). The solvent was evaporated in vacuum to dryness at $60{ }^{\circ} \mathrm{C}$, then dissolved in $200 \mu \mathrm{L}$ deionized water and used for derivatization.

The samples of $200 \mu \mathrm{L}$ were mixed with $63 \mu \mathrm{L}$ OPA/ MPA (ortho-phthaldialdehyde/3-mercaptopropionic acid) reagent solution, incubated at $22^{\circ} \mathrm{C}$ for $10 \mathrm{~min}$, and then cooled down to $5{ }^{\circ} \mathrm{C}$. Samples of $10 \mu \mathrm{L}$ were injected into the chromatographic system consisting of a Waters 2695 Separations Module equipped with thermostable autosampler $\left(5^{\circ} \mathrm{C}\right)$ and column module $\left(35^{\circ} \mathrm{C}\right)$, a Waters 2745 Fluorescent detector (Waters Milford, MA, USA) and with C-18 $(4.6 \times 150 \mathrm{~mm}, 3.5 \mu \mathrm{m})$ column. Gradient elution at a flow rate of $1 \mathrm{~mL} / \mathrm{min}$ was applied using mobile phase A $\left(20 \mathrm{mmol} / \mathrm{L}\left(\mathrm{NH}_{4}\right)_{2} \mathrm{CO}_{3}\right.$ in water, $\mathrm{pH}$ adjusted $7.50 \pm 0.05$ ) and mobile phase $\mathrm{B}$ (acetonitrile). The gradient condition was as follows: $0-13 \mathrm{~min}$ : $91 \% \mathrm{~A}$ and $9 \% \mathrm{~B} ; 13-15$ min: linear change to $70 \% \mathrm{~A}$ and $30 \% \mathrm{~B}$ and hold this setting for additional $5 \mathrm{~min} ; 20-20.1 \mathrm{~min}$ : linear change to $91 \% \mathrm{~A}$ and $9 \% \mathrm{~B}$ and hold until $25 \mathrm{~min}$.

Analytes were detected at $\lambda_{\text {ex }}=337 \mathrm{~nm}, \lambda_{\text {em }}=520 \mathrm{~nm}$ was used for arginine and homoarginine and $\lambda_{\mathrm{em}}=454 \mathrm{~nm}$ for ADMA and SDMA. Every patient had their serum sample analyzed $(\mathrm{n}=154)$.

\section{St. George's Respiratory questionnaire (SGRQ)}

The validated Hungarian version of SGRQ was delivered according to the SGRQ manual supplied by the copyright holder [33]. This version is validated for a 1-month recall period related to the patients' recollection of their symptoms. SGRQ was used to determine health impairment in patients by providing three component scores and a total score. The Symptoms score characterizes the effect, frequency and severity of respiratory symptoms over the preceding 1 month. It is reflective of the patients' perception of their recent respiratory problems. The Activity score quantifies disturbances in daily physical activity caused to patients, whereas the Impacts score covers a wide range of disturbances related to psycho-social function. The Total score summarizes the significance of the disease on overall health status. Scores are expressed as a percentage with 100 representing the worst possible health status and 0 indicating the best one. Differences in scores were considered clinically meaningful if they exceeded 4 percent points [34]. Every patient filled out the questionnaire $(\mathrm{n}=154)$ by means of supervised selfadministration. Data entry was performed by two independent raters. Each used the SGRQ calculator, and coded all positive responses as 1 and all negative responses as 0 . Where data were missing, cells were left blank. Data entry guidelines were diligently followed. Inter-rater variability assessed by Spearman correlation was $0.976(\mathrm{p}<0.001)$, $0.997(\mathrm{p}<0.001), 0.998(\mathrm{p}<0.001)$ and $0.998(\mathrm{p}<0.001)$ for the Symptoms, Activity, Impacts and Total scores of SGRQ, respectively. The mean of scores obtained by the independent raters was used for statistical analysis.

In addition, answers to Question 4 (Over the last 4 weeks, I have had attacks of wheezing) was used to assess the level of symptom control of asthma patients. Answers to this question were dichotomized as follows. Patients were considered well-controlled if they responded with yes to the options "not at all/only with chest infections". Dichotomized response to Question 4 was used for stratification of data to allow further analysis of the final multiple regression model.

\section{Statistical analysis}

Normality of distribution for continuous variables was checked with the Shapiro-Wilk test. If distribution was normal, Student's t test was used for the comparison of two data sets, if not, Mann-Whitney $U$ test was performed. Frequencies were compared with Pearson's $x^{2}$ test.

Demographic, anthropometric, anamnestic, laboratory and SGRQ data were compared regarding the lower or higher extent of airflow limitation using $\mathrm{R}_{\mathrm{aw}}$ values below $0.22 \mathrm{kPa} / \mathrm{L}$ as cutoff. (Please note that this cutoff is considered as the cutoff for normal by the manufacturer of the plethysmograph used as well as by others [35], but it is coincidentally equivalent to the median value for Raw in our sample). Furthermore, demographic, anthropometric, pulmonary function and SGRQ data were compared in terms of the presence (well-controlled) or absence (not well-controlled) of adequate symptom control indicated by the response to Question 4 of SGRQ.

The correlation of $R_{a w}$ and ADMA was established using Spearman's correlation (because data sets did not follow Gaussian distributions). In order to account for potential confounders, multiple regression modeling was carried out. This procedure was initiated by assessing normality of each variable. Values of CK, HDL-cholesterol, Apo B, B12 vitamin, folate, sTSH, ADMA, SDMA, L-arginine, $R_{a w}$ and $G_{a w}$ were log-transformed, furthermore reciprocal of urea and reciprocal of square of glucose concentration were computed to ensure Gaussian distribution of variables prior to linear regression. 
Simple linear regression analysis was performed with possible determinants of $\mathrm{R}_{\mathrm{aw}}$ and ADMA including traditional confounding factors (age, gender, height, disease duration in years) and indices descriptive of pulmonary function (mentioned above). Missing data were omitted. Furthermore, laboratory parameters descriptive of carbohydrate, lipid and arginine homeostasis, hepatic, kidney and skeletal muscle function as well as inflammation were assessed. After univariate testing, age and gender (as a priori variables) as well as all significant regressors were introduced into a multiple linear regression model to further quantify the relationship between airflow limitation (characterized by $R_{a w}$ as the outcome variable) and serum ADMA concentration. (Inclusion of the defined daily dose of corticosteroids into the initial model should be noted.) Variables were entered in the model simultaneously, and then factors not significantly contributing to the model were deleted (eventually, the final model contained all variables identified a priori, FEF25-75\% \% pred, and the Activity and Total scores of SGRQ). In addition, the final model was stratified with respect to the presence or absence of asthma control indicated by responses to Question 4 of SGRQ. Heteroskedasticity of the model was assessed with Cook-Weisberg test.

Statistical analysis was performed with Stata 13.0 software (Stata Corporation). Values are given as mean \pm SD or medians (with interquartile ranges: IQR), excepting regression coefficients which are presented with their $95 \%$ confidence intervals.

\section{Results}

\section{Patients}

The treatment history of the 154 asthma patients included in our study were as follows: 4 patients were treatment naïve at the time of inclusion; 3 patients received a fixed combination of ipratropium with fenoterol; 45 patients were treated with short-acting beta agonists (43 of them with an inhaled corticosteroid); 146 patients received inhaled corticosteroid as a mono-component preparation $(\mathrm{n}=18)$ or in fixed combination with long-acting and/ or short-acting beta agonists $(\mathrm{n}=128)$. Other medications included inhalational use of anticholinergic agents $(n=38)$, oral use of methylxanthines $(n=16)$, montelukast $(\mathrm{n}=35)$ and omalizumab subcutaneously $(\mathrm{n}=8)$. In summary, most asthma patients received inhaled corticosteroids (and if not, it was always due to co-morbidities rendering the risks related to corticosteroid therapy higher than the accrued benefits).

\section{Comparison of patients with lower and higher airway resistance}

Dichotomization of the patient population by $R_{a w}$ (using the cutoff of $R_{a w}<0.22$ for lower airway resistance) yielded two patient populations ( $\mathrm{n}=77$ and $\mathrm{n}=77)$ being homogenous with respect to most of parameters investigated (Table 1). Nevertheless, distribution of men and women differed as 42 and 28 men were present in the group without and with airflow limitation, respectively $(\mathrm{p}=0.023)$. In addition, the patient's height was slightly but statistically significantly smaller in the group showing increased $R_{a w}$, while duration of asthma, serum ADMA level and all SGRQ components and the total scores were significantly greater in the group with elevated $R_{a w}$. Dyslipidemia was also more frequent among patients with higher $\mathrm{R}_{\mathrm{aw}}$ (Table 1 ).

\section{Comparison of the well-controlled and not well-controlled patients}

Dichotomy of patients with regards to the response to Question 4 of SGRQ provided a well-controlled $(\mathrm{n}=123)$ and a not well-controlled $(\mathrm{n}=31)$ stratum (Table 2). Demographic and anthropometric characteristics, furthermore static pulmonary function parameters (volumes) did not differ significantly when compared these two groups (with the exception of height that was slightly but significantly smaller in the not well-controlled group, similarly to the group with elevated $R_{a w}$ ). Dynamic lung function parameters characterizing the flow in the airways were significantly smaller in the not well-controlled group, while there was no significant difference between the two groups with regard to objective measures of airway obstruction (e.g. $R_{a w}$ and its $G_{a w}$ ). All SGRQ scores were significantly higher in the not well-controlled group (indicating greater health impairment and poorer quality of life) (Table 2).

\section{Significant predictors of airway resistance and serum ADMA concentration}

The (log transformed) $R_{a w}$ showed positive correlation with (log transformed) ADMA upon the analysis of the whole data set (Spearman correlation coefficient: 0.27, $\mathrm{p}<0.001$ ) (Fig. 2) and of data obtained from the well-controlled asthma stratum (Spearman correlation coefficient: $0.30, \mathrm{p}<0.001$ ). In contrast, no statistically significant correlation was found in the not well-controlled asthma stratum (Spearman correlation coefficient: 0.12, $\mathrm{p}=0.51$ ).

Consistently, the simple linear regression (used to identify which parameters determine $R_{a w}$ and serum ADMA concentration) has proved that $\mathrm{R}_{\mathrm{aw}}$ and ADMA are mutually significant predictors for each other (Table 3 ). It is interesting to note that FEV1\% predicted, a parameter commonly used in clinical practice to characterize airway function showed significant linear association with (log) ADMA ( $\beta$ : $-0.0035, \mathrm{CI}-0.0067,-0.00,020 ; \mathrm{p}=0.01$ ). The positive association seen between $(\log ) R_{a w}$ and $(\log )$ ADMA remained statistically significant after multiple linear regression ( $\beta: 0.22$, CI: $0.054,0.383 ; p=0.01$ ), even after adjusting for all significant predictors and 
Table 1 Some characteristics of the whole population of bronchial asthma patients $(n=154)$ and of its two strata dichotomized according to lower $(n=77)$ or higher $(n=77)$ airway resistance $\left(R_{a w}\right)$

\begin{tabular}{|c|c|c|c|c|}
\hline Parameters & Whole population & Lower $\mathrm{R}_{\mathrm{aw}}$ & Higher $\mathrm{R}_{\mathrm{aw}}$ & $\mathrm{p}$ \\
\hline Age (years) & $49.00(36.00-58.00)$ & $44.00(36.00-57.00)$ & $52.00(41.00-59.00)$ & 0.086 \\
\hline Gender (f/m) & $84 / 70$ & $35 / 42$ & $49 / 28$ & 0.023 \\
\hline Smoker (n/y) & $134 / 20$ & $66 / 11$ & $68 / 9$ & 0.632 \\
\hline Smoking (pack-years) & $0.00(0.00-3.88)$ & $0.00(0.00-4.00)$ & $0.00(0.00-3.75)$ & 0.987 \\
\hline Diabetes $(n / y)$ & $147 / 7$ & $76 / 1$ & $71 / 6$ & 0.053 \\
\hline Dyslipidemia (n/y) & $108 / 46$ & $63 / 14$ & $45 / 32$ & 0.002 \\
\hline RR systolic (mmHg) & $132.10 \pm 15.08$ & $132.93 \pm 15.46$ & $131.23 \pm 14.73$ & 0.487 \\
\hline RR diastolic (mmHg) & $84.76 \pm 10.70$ & $85.80 \pm 9.75$ & $83.73 \pm 11.53$ & 0.231 \\
\hline Hypertension (n/y) & $95 / 59$ & $53 / 24$ & $42 / 35$ & 0.068 \\
\hline Disease duration (years) & $15.00(10.00-20.00)$ & $14.00(8.00-19.00)$ & $16.00(12.00-21.00)$ & 0.032 \\
\hline Waist (cm) & $96.49 \pm 12.87$ & $96.02 \pm 13.51$ & $96.97 \pm 12.28$ & 0.654 \\
\hline Weight (kg) & $75.00(65.00-88.00)$ & $76.00(66.00-89.50)$ & $75.00(65.00-87.00)$ & 0.632 \\
\hline Height (m) & $1.68 \pm 0.10$ & $1.70 \pm 0.10$ & $1.66 \pm 0.09$ & 0.004 \\
\hline $\mathrm{BMI}\left(\mathrm{kg} / \mathrm{m}^{2}\right)$ & $27.16 \pm 4.48$ & $26.61 \pm 4.56$ & $27.72 \pm 4.36$ & 0.127 \\
\hline ADMA $(\mu \mathrm{mol} / \mathrm{L})$ & $0.54(0.44-0.67)$ & $0.53(0.40-0.65)$ & $0.57(0.47-0.71)$ & 0.034 \\
\hline $\mathrm{SDMA}(\mu \mathrm{mol} / \mathrm{L})$ & $0.45(0.38-0.53)$ & $0.45(0.36-0.52)$ & $0.47(0.41-0.55)$ & 0.080 \\
\hline L-arginine $(\mu \mathrm{mol} / \mathrm{L})$ & $103.17(90.24-125.84)$ & $101.32(87.65-117.68)$ & 104.58 (91.70-129.90) & 0.209 \\
\hline B12 (pmol/L) & $322.25(237.30-398.10)$ & $321.00(228.80-390.70)$ & 323.50 (239.20-401.10) & 0.432 \\
\hline Folate (nmol/L) & 19.00 (15.19-23.99) & $19.50(14.76-24.15)$ & $18.91(16.00-23.16)$ & 0.779 \\
\hline Urea (mmol/L) & $4.50(3.90-5.50)$ & $4.30(3.90-5.30)$ & $4.60(3.90-5.55)$ & 0.667 \\
\hline Creatinine ( $\mu \mathrm{mol} / \mathrm{L})$ & $69.00(59.00-80.00)$ & $70.00(60.00-79.00)$ & $69.00(57.00-80.00)$ & 0.762 \\
\hline GFR $\left(\mathrm{ml} / \mathrm{min} / 1.73 \mathrm{~m}^{2}\right)$ & $91.00(85.00-91.00)$ & $91.00(87.00-91.00)$ & $91.00(85.00-91.00)$ & 0.469 \\
\hline GOT (U/L) & $20.00(16.00-25.00)$ & $21.00(17.00-24.00)$ & $19.00(16.00-25.50)$ & 0.314 \\
\hline GPT (U/L) & $19.00(15.00-28.00)$ & $19.00(15.00-27.00)$ & $18.00(14.00-29.00)$ & 0.314 \\
\hline$\gamma \mathrm{GT}(\mathrm{U} / \mathrm{L})$ & $23.00(16.00-34.00)$ & $23.00(16.00-32.00)$ & $22.50(15.50-34.50)$ & 0.920 \\
\hline CK (U/L) & $110.00(81.00-158.00)$ & $114.00(88.00-167.00)$ & $106.50(75.50-152.00)$ & 0.090 \\
\hline $\mathrm{LDH}(\mathrm{U} / \mathrm{L})$ & $196.00(180.00-223.00)$ & $195.00(180.00-224.00)$ & 197.50 (179.00-222.00) & 0.775 \\
\hline Glucose (mmol/L) & $5.00(4.30-5.50)$ & $5.00(4.20-5.40)$ & $5.00(4.50-5.50)$ & 0.388 \\
\hline Insulin (mU/L) & $9.05(6.25-17.35)$ & $9.00(6.40-16.50)$ & $9.50(6.00-17.70)$ & 0.569 \\
\hline $\operatorname{HgA1C}(\%)$ & $5.40(5.00-5.80)$ & $5.40(5.00-5.70)$ & $5.50(5.00-5.80)$ & 0.266 \\
\hline HOMA & $2.01(1.28-4.11)$ & $1.99(1.24-3.38)$ & $2.19(1.35-4.25)$ & 0.405 \\
\hline Cholesterol (mmol/L) & $5.32 \pm 1.20$ & $5.19 \pm 1.04$ & $5.44 \pm 1.33$ & 0.208 \\
\hline LDL-C (mmol/L) & $3.18 \pm 0.94$ & $3.08 \pm 0.86$ & $3.27 \pm 1.02$ & 0.230 \\
\hline $\mathrm{HDL}-\mathrm{C}(\mathrm{mmol} / \mathrm{L})$ & $1.40(1.20-1.75)$ & $1.40(1.20-1.70)$ & $1.40(1.20-1.90)$ & 0.429 \\
\hline Apo-A1 (g/L) & $1.58 \pm 0.29$ & $1.56 \pm 0.25$ & $1.60 \pm 0.32$ & 0.393 \\
\hline ApoB (g/L) & $0.99(0.85-1.18)$ & $0.95(0.85-1.08)$ & $1.00(0.85-1.22)$ & 0.320 \\
\hline $\mathrm{Lp}(\mathrm{a})(\mathrm{mg} / \mathrm{L})$ & $121.00(55.00-352.00)$ & $117.00(52.00-346.00)$ & $132.00(56.00-364.00)$ & 0.913 \\
\hline $\mathrm{TG}(\mathrm{mmol} / \mathrm{L})$ & $1.30(1.00-2.00)$ & $1.30(0.90-2.00)$ & $1.45(1.00-1.95)$ & 0.969 \\
\hline CRP (high/low) & $28 / 125$ & $13 / 64$ & $15 / 61$ & 0.648 \\
\hline Fibrinogen (g/L) & $3.35 \pm 0.64$ & $3.30 \pm 0.55$ & $3.39 \pm 0.72$ & 0.422 \\
\hline Procalcitonin $(\mu \mathrm{g} / \mathrm{L})$ & $0.00(0.00-0.00)$ & $0.00(0.00-0.00)$ & $0.00(0.00-0.00)$ & 0.317 \\
\hline SGRQ Symptoms score & $29.97(13.47-52.33)$ & $23.04(11.01-43.24)$ & $33.83(14.56-55.63)$ & 0.034 \\
\hline SGRQ Impacts score & $24.60(10.74-40.01)$ & $16.10(7.39-34.92)$ & 30.99 (20.50-48.05) & $<0.001$ \\
\hline SGRQ Activity score & $47.68(29.49-60.25)$ & $35.47(17.31-54.32)$ & $55.62(41.70-66.18)$ & $<0.001$ \\
\hline SGRQ Total score & $32.75(17.52-48.73)$ & $25.26(13.90-41.00)$ & $37.93(29.53-54.01)$ & $<0.001$ \\
\hline
\end{tabular}

The cutoff for $R_{a w}$ was $0.22 \mathrm{kPa} s / \mathrm{L}$, with $<0.22$ and $\geq 0.22 \mathrm{kPa} / \mathrm{L}$ indicating lower $(\mathrm{n}=77)$ and higher $(\mathrm{n}=77)$ airway resistance, respectively. Data are presented as mean $\pm \mathrm{SD}$ or median (interquartile range) unless otherwise stated $S G R Q$ St. George's Respiratory questionnaire

Differences between the two groups were considered significant at $\mathrm{p}<0.05$ (indicated in italics) 
Table 2 Main characteristics of the whole population of bronchial asthma patients $(n=154)$ and of its two strata divided on the basis of the response to Question 4 of SGRQ, i.e. well-controlled $(n=123)$ and not well-controlled $(n=31)$ groups

\begin{tabular}{|c|c|c|c|c|}
\hline Parameters & Whole population & Well-controlled & Not well-controlled & $\mathrm{p}$ \\
\hline \multicolumn{5}{|c|}{ Demographic and anthropometric parameters } \\
\hline Age (years) & $49.00(36.00-58.00)$ & $48.00(36.00-59.00)$ & $52.00(40.00-56.00)$ & 0.442 \\
\hline Gender (f/m) & $84 / 70$ & $63 / 60$ & $21 / 10$ & 0.099 \\
\hline Height (m) & $1.68 \pm 0.098$ & $1.69 \pm 0.097$ & $1.65 \pm 0.099$ & 0.034 \\
\hline Weight (kg) & $75.00(65.00-88.00)$ & $76.00(67.00-88.00)$ & $72.00(62.00-87.00)$ & 0.278 \\
\hline \multicolumn{5}{|c|}{ Lung function parameters } \\
\hline FVC\% pred & $92.55 \pm 13.80$ & $93.45 \pm 13.60$ & $89.00 \pm 14.25$ & 0.101 \\
\hline IVC\% pred & $97.50(87.00-106.00)$ & $98.00(88.00-106.00)$ & $97.00(81.00-102.00)$ & 0.267 \\
\hline TLC\% pred & $108.65 \pm 13.78$ & $108.25 \pm 14.27$ & $110.26 \pm 11.75$ & 0.470 \\
\hline TGV\% pred & $125.25 \pm 22.11$ & $124.62 \pm 22.18$ & $127.77 \pm 21.99$ & 0.479 \\
\hline RV\% pred & $132.50(117.00-157.00)$ & 132.00 (117.00-152.00) & 143.00 (114.00-175.00) & 0.266 \\
\hline RV/TLC \% pred & $123.84 \pm 20.06$ & $122.41 \pm 19.84$ & $129.48 \pm 20.24$ & 0.079 \\
\hline FEV1\% pred & $86.10 \pm 15.07$ & $87.99 \pm 13.97$ & $78.58 \pm 17.08$ & 0.002 \\
\hline PEF\% pred & $75.14 \pm 18.21$ & $78.80 \pm 16.12$ & $60.61 \pm 18.98$ & $<0.001$ \\
\hline FEF25-75\% \% pred & $66.54 \pm 22.22$ & $69.87 \pm 21.58$ & $53.32 \pm 19.98$ & $<0.001$ \\
\hline MEF50\% \% pred & $68.81 \pm 24.94$ & $72.36 \pm 23.85$ & $54.74 \pm 24.58$ & $<0.001$ \\
\hline FEV1/IVC\% pred & $90.92 \pm 10.51$ & $92.41 \pm 10.23$ & $85.00 \pm 9.61$ & $<0.001$ \\
\hline FEV1/FVC & $0.77(0.73-0.83)$ & $0.79(0.74-0.84)$ & $0.74(0.68-0.78)$ & 0.002 \\
\hline $\mathrm{R}_{\mathrm{aw}}(\mathrm{kPa} \mathrm{s} / \mathrm{L})$ & $0.22(0.18-0.29)$ & $0.21(0.17-0.28)$ & $0.25(0.19-0.31)$ & 0.152 \\
\hline $\mathrm{G}_{\mathrm{aw}}(\mathrm{L} / \mathrm{kPa} \mathrm{s})$ & $4.67(3.44-5.55)$ & $4.78(3.55-5.73)$ & $3.94(3.2-5.35)$ & 0.149 \\
\hline \multicolumn{5}{|l|}{ SGRQ } \\
\hline Symptoms score & $29.97(13.47-52.33)$ & $22.44(11.01-37.28)$ & $64.49(51.75-74.89)$ & $<0.001$ \\
\hline Impacts score & $24.60(10.74-40.01)$ & $20.50(8.58-34.92)$ & $42.59(32.10-54.24)$ & $<0.001$ \\
\hline Activity score & $47.68(29.49-60.25)$ & $41.76(23.33-59.45)$ & $59.45(53.01-72.85)$ & $<0.001$ \\
\hline Total score & $32.75(17.52-48.73)$ & $28.35(16.22-41.55)$ & $53.71(41.22-60.93)$ & $<0.001$ \\
\hline
\end{tabular}

Data are presented as mean $\pm S D$ or median (interquartile range) unless otherwise stated

SGRQ St. George's Respiratory questionnaire

Differences between the two groups were considered significant at $\mathrm{p}<0.05$ (indicated in italics)

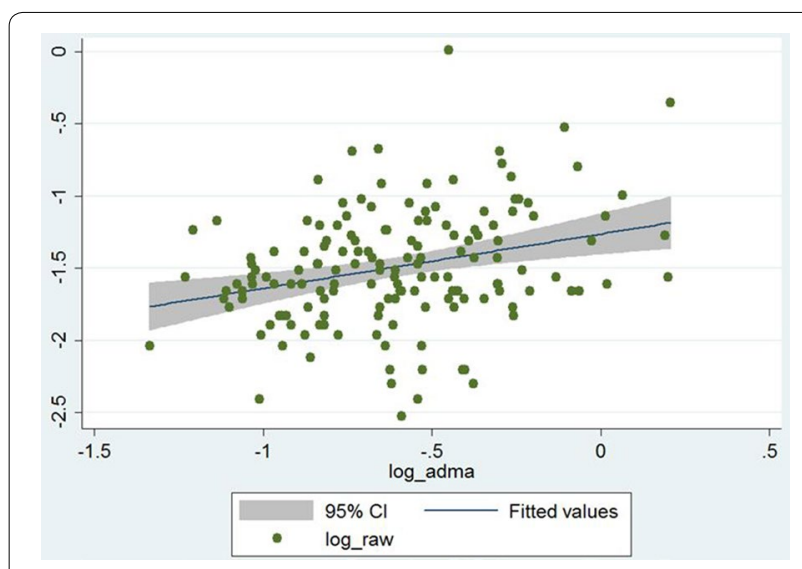

Fig. 2 Correlation of airway resistance $\left(R_{a w}\right)$ and asymmetric dimethylarginine (ADMA) serum levels in the whole data set $(n=154)$. The grey zone indicates the $95 \%$ confidence interval, while the blue line (in it) shows the fitted values of $\log$ ADMA and $\log R_{a w}$ data pairs determinants identified in advance (Table 3). The positive association between $(\log ) \mathrm{R}_{\mathrm{aw}}$ and (log) ADMA became even more pronounced in the stratum of well-controlled asthma patients ( $\beta: 0.25, \mathrm{CI}: 0.08,0.41 ; \mathrm{p}=0.005)$, while there was a weak, statistically not significant association in the not well-controlled stratum ( $\beta$ : 0.14, CI: -0.40 , $0.67 ; \mathrm{p}=0.60)$. The final model and its stratified models showed no heteroskedasticity $(\mathrm{p}=0.57, \mathrm{p}=0.78$ and $p=0.19$ for the full model, well-controlled and not wellcontrolled strata, respectively). The final model showed good fit reflected by the Cook-Weisberg test $(\mathrm{p}=0.57)$ and by locally weighted scatterplot smoothing (Fig. 3).

In addition, the final model (for $\mathrm{R}_{\mathrm{aw}}$ ) indicated a negative association with FEF25-75\% \% pred, a lung function parameter descriptive of small airway dysfunction and a positive correlation with the Activity score of SGRQ reflective of the disturbance the patient suffers with respect to daily physical activity. Surprisingly, in the final 
Table 3 Significant (and two almost significant) predictors of asymmetric dimethylarginine (ADMA) serum level and airway resistance $\left(R_{a w}\right)$ determined with simple and multiple (for $\mathbf{R}_{\mathrm{aw}}$ only) linear regression for the whole population of bronchial asthma patients $(n=154)$

\begin{tabular}{|c|c|c|}
\hline Parameter & Coefficient $(95 \% \mathrm{Cl})$ & $\mathrm{p}$ \\
\hline \multicolumn{3}{|c|}{ Simple linear regression of ADMA (log transformed) } \\
\hline Height & $-0.75(-1.25,-0.26)$ & 0.003 \\
\hline Disease duration & $0.0061(0.00062,0.012)$ & 0.029 \\
\hline $\log$ L-arginine & $0.27(0.083,0.45)$ & 0.005 \\
\hline $\log$ SDMA & $0.77(0.62,0.93)$ & $<0.001$ \\
\hline $\log \mathrm{TG}$ & $0.091(0.0022,0.18)$ & 0.045 \\
\hline GFR (normal/low) & $0.12(0.022,0.23)$ & 0.018 \\
\hline FVC\% pred & $-0.0037(-0.0072,0.00010)$ & 0.044 \\
\hline FEV1\% pred & $-0.0035(-0.0067,-0.00020)$ & 0.038 \\
\hline FEF25-75\% \% pred & $-0.0022(-0.0044,0.000013)$ & 0.051 \\
\hline $\log R_{a w}$ & $0.22(0.10,0.34)$ & $<0.001$ \\
\hline $\log G_{a w}$ & $-0.22(-0.33,-0.10)$ & $<0.001$ \\
\hline \multicolumn{3}{|c|}{ Simple linear regression of $\mathrm{R}_{\mathrm{aw}}$ (log transformed) } \\
\hline Age & $0.0046(0.00019,0.0090)$ & 0.041 \\
\hline Gender & $-0.20(-0.33,-0.075)$ & 0.002 \\
\hline Height & $-1.22(-1.86,-0.58)$ & $<0.001$ \\
\hline Disease duration & $0.0077(0.00051,0.015)$ & 0.036 \\
\hline Dyslipidemia & $0.18(0.043,0.32)$ & 0.011 \\
\hline Hypertension & $0.19(0.059,0.32)$ & 0.005 \\
\hline BMI & $0.014-0.000066,0.029)$ & 0.051 \\
\hline Albumin & $-0.028(-0.051,-0.0052)$ & 0.017 \\
\hline $\log A D M A$ & $0.38(0.17,0.58)$ & $<0.001$ \\
\hline $\log$ SDMA & $0.29(0.031,0.54)$ & 0.028 \\
\hline FVC\% pred & $-0.010(-0.015,-0.0059)$ & $<0.001$ \\
\hline FEV1\% pred & $-0.14(-0.017,-0.0099)$ & $<0.001$ \\
\hline FEV1/FVC & $-0.018(-0.0263,-0.011)$ & $<0.001$ \\
\hline FEF25-75\% \% pred & $-0.15(-0.24,-0.062)$ & 0.001 \\
\hline RV\% pred & $0.0022(0.00033,0.0042)$ & 0.022 \\
\hline RV/TLC\% pred & $0.0068(0.0037,0.0098)$ & $<0.001$ \\
\hline IC/TLC & $-1.22(-1.95,-0.45)$ & 0.001 \\
\hline SGRQ activity score & $0.0071(0.0044,0.0097)$ & $<0.001$ \\
\hline SGRQ impacts score & $0.0044(0.00094,0.0079)$ & 0.013 \\
\hline SGRQ total score & $0.0059(0.0025,0.0093)$ & 0.001 \\
\hline \multicolumn{3}{|c|}{ Multiple linear regression of $R_{a w}$ (log transformed) } \\
\hline $\log A D M A$ & $0.22(0.054,0.383)$ & 0.01 \\
\hline FEF25-75\% \% pred & $-0.009(-0.01,-0.006)$ & $<0.001$ \\
\hline SGRQ activity score & $0.009(0.004,0.014)$ & $<0.001$ \\
\hline SGRQ total score & $-0.007(-0.012,-0.001)$ & 0.019 \\
\hline
\end{tabular}

Statistically significant $p$ values are indicated in italic $(p<0.05)$

Regression coefficient values are presented with their $95 \%$ confidence limits

Significant parameters provided by the simple regression (together with the relevant a priori identified parameters) served as an initial model for the multiple regression analysis

model, $R_{a w}$ is negatively associated with the global effect disease has on the patient's well-being (reflected by the Total score of SGRQ) (Table 3).

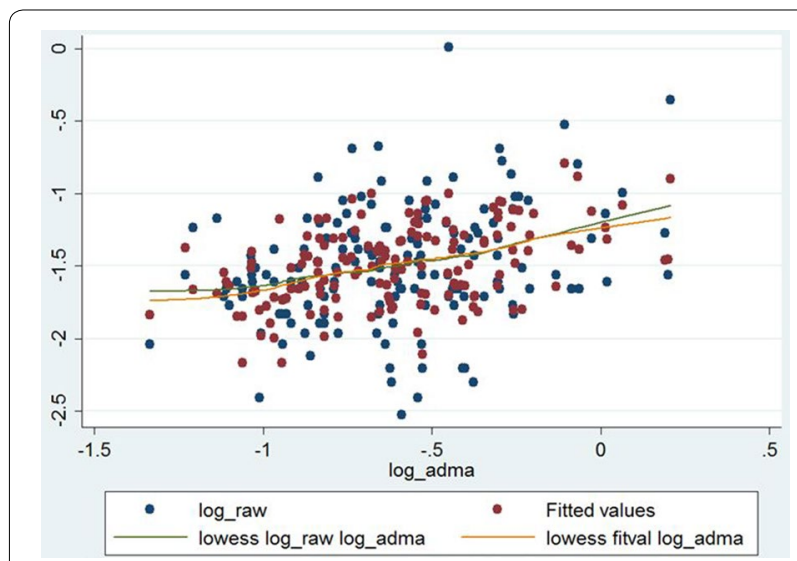

Fig. 3 The model of correlation of airway resistance $\left(R_{a w}\right)$ and asymmetric dimethylarginine (ADMA) serum concentration. The blue dots indicate the raw (i.e. original) data, while the red dots indicate the fitted values obtained by multiple linear regression. The green and orange lines indicate the fitted curves for raw data and for data provided by multiple regression. The fitted curves were obtained by locally weighted scatterplot smoothing (lowess)

Further assessment of the final model exhibited that $(\log ) \mathrm{R}_{\mathrm{aw}}$ showed good correlation with FEF25-75\% \% pred in the whole, well-controlled and not well-controlled strata (Spearman correlation coefficient: -0.53 , -0.54 and $-0.39, \mathrm{p}<0.001, \mathrm{p}<0.001$ and $\mathrm{p}=0.027$, respectively). Consistently, there was a strong, significant positive correlation between $(\log ) G_{a w}$ and FEF25-75\% \% pred in the whole sample, in the well-controlled and in the not well-controlled stratum too (Spearman correlation coefficient: $0.53,0.54$ and $0.39, \mathrm{p}<0.001, \mathrm{p}<0.001$ and $p=0.031$, respectively). In addition, total score of SGRQ showed a significant negative correlation with FEV1\% pred (Spearman correlation coefficient: -0.33 , $\mathrm{p}<0.001$ ), which correlation also remained significant in both the well-controlled and not well-controlled strata (Spearman correlation coefficient: -0.24 and -0.36 , $\mathrm{p}=0.007$ and $\mathrm{p}=0.049$, respectively). (Other parameters indicative of small airway disease such as RV\% pred and RV/TLC\% pred [36, 37], which were also included in the original model, did not contribute significantly to the model thus they were finally omitted.)

\section{Discussion}

The main finding of the current study is that serum ADMA shows significant positive correlation with airway limitation characterized by $\mathrm{R}_{\mathrm{aw}}$ in adult asthma patients receiving asthma controller therapy (including, for most patients, an inhaled corticosteroid). This relationship remained significant even after adjusting for potential confounders. Furthermore, this positive association was more pronounced when the analysis was restricted to 
patients attaining a high level of asthma control. Upon evaluating the inflammatory status of our patient cohort, we found that general markers of inflammation (CRP, procalcitonin and fibrinogen) are in the normal range and show no significant difference with respect to airflow limitation $\left(\mathrm{R}_{\mathrm{aw}}\right)$. This lack of evidence for systemic inflammation could be due to the fact that these patients all received control-based asthma therapy at the time of our investigations ( $94 \%$ of them $(n=146)$ were on inhaled corticosteroid therapy).

These findings have several implications regarding $\mathrm{L}$-arginine-NO homeostasis including the interaction between ADMA and distinct NOS isoforms. Prior reports demonstrated that use of even low-dose corticosteroids is able to inhibit the activation of NF- $\mathrm{kB}$ and the subsequent iNOS synthesis [12]. Moreover, glucocorticoids can decrease systemic inflammatory signals needed for inducing iNOS transcription [8]. Considering these effects together with the observation that ADMA seems to preferentially inhibit constitutive NO synthases [38], we postulate that, in our cohort (mainly in the wellcontrolled stratum), iNOS is not or minimally induced and thus its inhibition by ADMA is marginal. This means that the potential beneficial effect of ADMA conferred by inhibition of iNOS may be insignificant. Therefore, we suggest that, in our sample, inhibition of NOS is only evident with respect to the constitutive isoforms. As such, elevated levels of ADMA may elicit deleterious effects by decreasing NO produced by the constitutive NOS isoforms. Furthermore, ADMA, by uncoupling NOS, could lead to increased reactive oxygen and nitrogen species formation in airway epithelial cells [38]. Starting from the observation that ADMA enhanced arginase activity leading to collagen production [20], a reversible fibrosis provoked by ADMA (Fig. 1) may also contribute to the increase of $R_{a w}$. This concept is further supported by the weaker association seen between $R_{a w}$ and serum ADMA levels (indicated by the lower value of the regression coefficient and lack of statistical significance). Given the fact that presence of wheezing is possibly indicative of airway inflammation (being present regardless of the medication used), it may be presumed that these inflammatory processes induce iNOS. It would follow that inhibition of iNOS by ADMA in these patients (e.g. in the not well-controlled stratum) hence would counteract the deleterious effects exerted by iNOS and would manifest in a lower increase of $\mathrm{R}_{\mathrm{aw}}$.

The fact that significant difference was only evidenced for dynamic lung function parameters when patients were compared with respect to their level of symptom control (Table 2) may be due to fact that flow parameters (as well as the Tiffeneau index) compile information regarding airway resistance and respiratory effort. The latter is influenced by elastic recoil of the lung, respiratory muscle strength and stiffness of the chest. It is highly probable that those patients who experienced wheezing over the past 4 weeks are in a poorer general physical condition, further reflected by the significantly and clinically meaningful differences in the Symptoms, Activity and Impacts component scores as well as the totals score for SGRQ. So, it seems probable that the significant difference seen with respect to flow parameters is due to the difference in general physical state and consequent muscle strength of these two groups. This difference is absent in objective measures of airflow limitation, e.g. $R_{a w}$ and $G_{a w}$ as these measures are not influenced by respiratory effort.

Previously we have proposed that, in the range of normal concentrations $(0.35-1.0 \mu \mathrm{mol} / \mathrm{L})$, ADMA confers protection against atherosclerosis [6], a lesion where iNOS is induced, by causing a more pronounced inhibition on iNOS due to the lower $\mathrm{EC}_{50}$ value of ADMA for iNOS than that for eNOS $[39,40]$. Although, in the present study, serum ADMA levels were also in the normal range, this beneficial effect of ADMA was not seen possibly due to the inhibitory effect inhaled corticosteroids have on airway iNOS expression (which makes inhibition of iNOS by ADMA insignificant in asthma patients receiving corticosteroid therapy).

In general, few reports are available regarding the systemic level of ADMA in pediatric and adult asthma patients. Our ADMA values are comparable with results of others. In a recent study, systemic ADMA levels of $0.37 \mu \mathrm{mol} / \mathrm{L}$ (IQR: $0.29,0.59$ ) and $0.48 \mu \mathrm{mol} / \mathrm{L}$ (IQR: $0.35,0.7)$ were reported for adult patients suffering from early- and late-onset asthma, respectively [22]. Upon assessment of children with asthma, a group found serum ADMA levels corresponding to $0.53 \mu \mathrm{mol} / \mathrm{L}$ (CI: $0.47,0.6$ ) [41], while yet another group reported mean plasma ADMA concentration of $0.58 \pm 0.05 \mu \mathrm{mol} / \mathrm{L}$ [23]. Others described considerably higher circulating levels of ADMA in children suffering from asthma $(0.92 \pm 0.20 \mu \mathrm{mol} / \mathrm{L})$, however this showed no significant difference when compared to healthy controls also included in that study $(0.91 \pm 0.23 \mu \mathrm{mol} / \mathrm{L}, \mathrm{p}=0.88)$ [42].

Our finding that the final model describing the relationship between $R_{a w}$ and ADMA includes FEF25-75\% \% pred (Table 3) could be interpreted in view of the wellestablished notion that asthma compromises the function of the small airways [43]. This is especially true for airways with internal diameter lower than $2 \mathrm{~mm}$ [44]. In fact, small airways were implicated to be one of the chief sites of airflow limitation [45]. In line with these observations, we have found that FEF25-75\% \% pred shows a strong negative correlation with $R_{a w}$ implying 
the contribution of small airways to airflow limitation (accordingly, $\mathrm{G}_{\mathrm{aw}}$, the reciprocal of $\mathrm{R}_{\mathrm{aw}}$, exhibits a significant positive correlation with FEF $25-75 \% \%$ pred). The fact that this strong correlation proved to be significant irrespective of the level of asthma control achieved is interesting if one considers that targeting distal airways by means of an inhalational therapy remains a challenge [43]. Our finding that inflammation of small airways (reflected by decreased FEF25-75\% \% pred) parallels that of larger airways (indicated by increased $\mathrm{R}_{\mathrm{aw}}$ ) emphasizes that special attention must be paid to optimal delivery of inhalational medication in daily clinical practice to allow for sufficient relief of airflow limitation stemming from the distal airways.

The third set of parameters that contribute significantly to the explanation of the relationship between $R_{a w}$ and ADMA is related to the health impairment reported by asthma patients of our cohort. In agreement with prior studies [46], we have found a significant negative correlation between the Total score of SGRQ and FEV1\% pred. Furthermore, we found that Activity score shows significant positive correlation with $\mathrm{R}_{\mathrm{aw}}$, indicating that airway limitation is associated with loss of quality of life conferred by disturbance of physical activity. The negative association seen between the Impacts score and $R_{a w}$ in our cohort may be due to the correction of overrepresentation of the influence of Activity score due to the fact that it is included in the Total score as well (so, it might be an inherent feature of SGRQ). The clinically meaningful difference of the three components as well as the Total score compared between groups of lower and higher $R_{a w}$ values (Table 1 ) further emphasizes the deleterious effect of airway limitation on the quality of life of patients.

A limitation of the present study is the lack of direct evidence for the activity of distinct NOS isoforms (as specimens related to the different compartments). Thus, we only presume a suppressed iNOS activity starting from the absence of elevated inflammatory markers in the serum that makes the explanation of our findings somewhat speculative. Further limitation of the study relates to the lack of characterization of oxidative and nitrosative stress by means of determining stable end products (nitrite and nitrate). In addition, it must be acknowledged that Raw was not expressed as percent predicted (described by [47]) due to technical limitations, hence this measure was not adjusted for weight and height. Nevertheless, height and BMI identified as significant regressors for Raw and/or (log)ADMA (by the simple linear regression (Table 3)) were included in the initial multiple model. However, both of these parameters were omitted due to their lack of significant contribution to the final model. FEF25-75\% \% pred should be interpreted with caution because of difficulties with reproducibility previously reported by others [36]. Finally, it should be mentioned that our analysis does not address the timing of the measurements in relationship to the administration of bronchodilators or other medications, thus although patients were examined while being on maintenance therapy, timing of the medication consumption may induce a variability.

\section{Conclusions}

To the best of our knowledge, this is the first study in which the relationship was investigated between serum ADMA level and $\mathrm{R}_{\mathrm{aw}}$, an objective functional parameter characteristic of airflow limitation in asthma. The strong positive correlation between ADMA and $R_{a w}$ in a multiple linear regression model may indicate that ADMA contributes to the development of bronchoconstriction in asthma patients receiving control-based asthma therapy. This is further emphasized by the fact that this correlation became even stronger when the analysis was limited to the stratum of well-controlled patients. Based on the correlation between ADMA and $\mathrm{R}_{\mathrm{aw}}$, we shed light on the potential importance of ADMA in the pathomechanism of asthma. In addition, our results further demonstrate that $R_{a w}$ is a valuable parameter in the assessment of airflow limitation in asthma patients.

\section{Abbreviations}

ACOS: asthma-COPD overlap syndrome; ADMA: asymmetric dimethylarginine; apoA1: apolipoprotein A1; apoB: apolipoprotein B; AR: allergic rhinitis; ATS:

American Thoracic Society; BTPS: body temperature and pressure saturated; CK: creatine kinase; COPD: chronic obstructive pulmonary disease; CRP: $\mathrm{C}$-reactive protein; $\mathrm{DDAH}$ : dimethylargininase; $\mathrm{EC}_{50}$ : agonist concentration producing half maximal effect; eNOS: endothelial NOS; ERS: European Respiratory Society; FEF $25-75 \% \%$ pred: forced expiratory flow between 25 and $75 \%$ of FVC, percent of predicted value; FEV $1 \%$ pred: forced expiratory volume in $1 \mathrm{~s}$, percent of predicted value; FEV1/FVC: ratio of FEV1 to FVC $=$ FEV1\% $=$ Tiffeneau index; FEV1/IVC\% pred: ratio of FEV1 to IVC, percent of predicted value; FVC\% pred: forced vital capacity, percent of predicted value; $G_{\text {aw: }}$ airway conductance; GINA: Global Initiative for Asthma; GOT: glutamate-oxaloacetate transaminase; GPT: glutamate-pyruvate transaminase; HDL: high-density lipoprotein; HgA1c: hemoglobin A1c (glycated hemoglobin); HOMA: homeostasis model assessment; HPLC: high-performance liquid chromatography; IC/TLC: ratio of inspiratory capacity to total lung capacity; iNOS: inducible NOS; IQR: interquartile range; IVC\% pred: inspiratory vital capacity, percent of predicted value; LDH: lactate dehydrogenase; LDL: low-density lipoprotein; Lp(a): lipoprotein (a); MEF50\% \% pred: maximal expiratory flow at 50\% of FVC, percent of predicted value; MPA: 3-mercaptopropionic acid; NF-KB: nuclear factor kappa-light-chain-enhancer of activated B cells; nNOS: neuronal NOS; NO: nitric-oxide; NOHA: N-hydroxyarginine; NOS: nitric-oxide synthase; $\mathrm{O}^{2-}$ : superoxide anion; $\mathrm{ONOO}^{-}$: peroxynitrite anion; OPA: ortho-phthalaldehyde; PEF\% pred: peak expiratory flow, percent of predicted value; PRMT: protein methyltransferase; $R_{a w}$ : airway resistance; $R V \%$ pred: residual volume, percent of predicted value; RV/TLC\% pred: ratio of RV to TLC, percent of predicted value; SD: standard deviation; SDMA: symmetrical dimethylarginine; SGRQ: St. George's Respiratory questionnaire; STROBE: strengthening the reporting of observational studies in epidemiology; STSH: thyroid-stimulating hormone determined in a sensitive manner; TGV\% pred: thoracic gas volume, percent of predicted value; TLC\% pred: total lung capacity, percent of predicted value; үGT: gamma-glutamyl transferase. 


\begin{abstract}
Authors' contributions
PK, MA, FA, PC and SME participated in the patient recruitment and collection of clinical data. KL helped to perform the statistical analysis. TG and KS carried out the ADMA, SDMA and arginine measurement. GR participated in the design of the study and helped to draft the manuscript. SM coordinated the patient recruitment and clinical data collection. ZJ conceived of the study, performed the statistical analysis and completed the manuscript. All authors read and approved the final manuscript.
\end{abstract}

\section{Author details}

${ }^{1}$ Department of Health Systems Management and Quality Management for Health Care, Faculty of Public Health, University of Debrecen, Nagyerdei krt. 98, Debrecen 4032, Hungary. ${ }^{2}$ Institute of Clinical Pharmacology, Infectious Diseases and Allergology, Kenezy Gyula Teaching County Hospital and Outpatient Clinic, Bartok Bela ut 2-26, Debrecen 4031, Hungary. ${ }^{3}$ Department of Applied Chemistry, Faculty of Science and Technology, University of Debrecen, Egyetem ter 1, Debrecen 4032, Hungary. ${ }^{4}$ Department of Pharmacology and Pharmacotherapy, Faculty of Medicine, University of Debrecen, Nagyerdei krt 98, Debrecen 4032, Hungary. ${ }^{5}$ Department of Pulmonology, Faculty of Medicine, University of Debrecen, Nagyerdei krt. 98, Debrecen 4032, Hungary.

\section{Acknowledgements}

The authors acknowledge the technical assistance of Anett Lepp for the preparation of the manuscript.

\section{Competing interests}

The authors declare that they have no competing interests.

\section{Availability of data and materials}

Database underlying results of the present study is being on further evaluation, thus it is not freely available yet.

\section{Consent for publication}

Not applicable.

\section{Ethics approval and consent to participate}

The present study was approved by the Ethical Committee of the University of Debrecen (DEOEC RKEB/IKEB 3632-2012), and was only commenced following this ethical approval. Informed consent was obtained from each participant. The investigation conforms to the principles outlined in the Declaration of Helsinki.

\section{Ethics approval for animal use}

Not applicable.

\section{Funding}

Our current research was supported by the Scientific Research Grant of the Hungarian Foundation for Pulmonology (awarded in 2015) and the Hungarian Brain Research Program (KTIA_13_NAP-A-V/2). The role of the funding body was to provide funding based on a predefined research proposal. The funding body had no role in the design of the study, collection, analysis and interpretation of data or writing the manuscript.

\section{Publisher's Note}

Springer Nature remains neutral with regard to jurisdictional claims in published maps and institutional affiliations.

Received: 15 March 2017 Accepted: 15 December 2017

Published online: 03 January 2018

\section{References}

1. To T, Stanojevic S, Moores G, Gershon AS, Bateman ED, Cruz AA, Boulet L. Global asthma prevalence in adults: findings from the cross-sectional world health survey. BMC Public Health. 2012;12:1.

2. Braman SS. The global burden of asthma. Chest Journal. 2006;130:4S-12S
3. Global Initiative for Asthma. Global strategy for asthma management and prevention. 2016. http://ginasthma.org/wp-content/uploads/2016/04/ wms-GINA-2016-main-report-final.pdf. Accessed 11 June 2017.

4. Vestbo J, Hurd SS, Agustí AG, Jones PW, Vogelmeier C, Anzueto A, Barnes PJ, Fabbri LM, Martinez FJ, Nishimura M. Global strategy for the diagnosis, management, and prevention of chronic obstructive pulmonary disease: GOLD executive summary. Am J Respir Crit Care Med. 2013;187:347-65.

5. Zsuga J, Torok J, Magyar MT, Valikovics A, Gesztelyi R, Keki S, Csiba L, Zsuga M, Bereczki D. Serum asymmetric dimethylarginine negatively correlates with intima-media thickness in early-onset atherosclerosis. Cerebrovasc Dis. 2007;23:388-94.

6. Zsuga J, Török J, Magyar MT, Valikovics A, Gesztelyi R, Lenkei Á, Csiba L, Kéki S, Zsuga M, Bereczki D. Dimethylarginines at the crossroad of insulin resistance and atherosclerosis. Metab Clin Exp. 2007;56:394-9.

7. Dupont LL, Glynos C, Bracke KR, Brouckaert P, Brusselle GG. Role of the nitric oxide-soluble guanylyl cyclase pathway in obstructive airway diseases. Pulm Pharmacol Ther. 2014;29:1-6.

8. Ricciardolo FL, Nijkamp FP, Folkerts G. Nitric oxide synthase (NOS) as therapeutic target for asthma and chronic obstructive pulmonary disease. Curr Drug Targets. 2006;7:721-35.

9. Fischer A, Hoffmann B. Nitric oxide synthase in neurons and nerve fibers of lower airways and in vagal sensory ganglia of man. Correlation with neuropeptides. Am J Respir Crit Care Med. 1996;154:209-16.

10. Guo FH, De Raeve HR, Rice TW, Stuehr DJ, Thunnissen FB, Erzurum SC. Continuous nitric oxide synthesis by inducible nitric oxide synthase in normal human airway epithelium in vivo. Proc Natl Acad Sci U S A. 1995;92:7809-13.

11. Wells SM, Holian A. Asymmetric dimethylarginine induces oxidative and nitrosative stress in murine lung epithelial cells. Am J Respir Cell Mol Biol. 2007;36:520-8.

12. Haddad E, Liu SF, Salmon M, Robichaud A, Barnes PJ, Chung KF. Expression of inducible nitric oxide synthase mRNA in Brown Norway rats exposed to ozone: effect of dexamethasone. Eur J Pharmacol Environ Toxicol Pharm. 1995;293:287-90.

13. Prado CM, Martins MA, Tibério IF. Nitric oxide in asthma physiopathology. ISRN allergy. 2011;2011:832560.

14. Saleh D, Ernst P, Lim S, Barnes PJ, Giaid A. Increased formation of the potent oxidant peroxynitrite in the airways of asthmatic patients is associated with induction of nitric oxide synthase: effect of inhaled glucocorticoid. FASEB J. 1998;12:929-37.

15. Brindicci C, Ito K, Barnes PJ, Kharitonov SA. Effect of an inducible nitric oxide synthase inhibitor on differential flow-exhaled nitric oxide in asthmatic patients and healthy volunteers. CHEST J. 2007;132:581-8.

16. Klein E, Weigel J, Buford MC, Holian A, Wells SM. Asymmetric dimethylarginine potentiates lung inflammation in a mouse model of allergic asthma. Am J Physiol Lung Cell Mol Physiol. 2010;299:L816-25.

17. Cardounel AJ, Xia Y, Zweier JL. Endogenous methylarginines modulate superoxide as well as nitric oxide generation from neuronal nitric-oxide synthase: differences in the effects of monomethyl- and dimethylarginines in the presence and absence of tetrahydrobiopterin. J Biol Chem. 2005;280:7540-9.

18. Druhan LJ, Forbes SP, Pope AJ, Chen C, Zweier JL, Cardounel AJ. Regulation of eNOS-derived superoxide by endogenous methylarginines. Biochemistry (NY). 2008;47:7256-63.

19. Ahmad T, Mabalirajan U, Ghosh B, Agrawal A. Altered asymmetric dimethyl arginine metabolism in allergically inflamed mouse lungs. Am J Respir Cell Mol Biol. 2010;42:3-8.

20. Kinker KG, Gibson AM, Bass SA, Day BP, Deng J, Medvedovic M, Figueroa JAL, Hershey GKK, Chen W. Overexpression of dimethylarginine dimethylaminohydrolase 1 attenuates airway inflammation in a mouse model of asthma. PLoS ONE. 2014;9:e85148.

21. Calabrese C, Carpagnano GE, Patella V, Vatrella A, Santus P. Asymmetric dimethylarginine (ADMA): will be or will not be a new revolutionary biomarker of bronchial asthma? Minerva Med. 2015;106(5(suppl. 1)):9-11.

22. Holguin F, Comhair SA, Hazen SL, Powers RW, Khatri SS, Bleecker ER, Busse WW, Calhoun WJ, Castro M, Fitzpatrick AM. An association between L-arginine/asymmetric dimethyl arginine balance, obesity, and the age of asthma onset phenotype. Am J Respir Crit Care Med. 2013;187:153-9.

23. Riccioni G, Bucciarelli V, Verini M, Consilvio N, Gallina S, Martini F, Aceto A, Scotti L, Bucciarelli T. ADMA, SDMA, L-Arginine and nitric oxide in allergic pediatric bronchial asthma. J Biol Regul Homeost Agents. 2012;26:561. 
24. Von Elm E, Altman DG, Egger M, Pocock SJ, Gøtzsche PC, Vandenbroucke JP. Strobe Initiative. The strengthening the reporting of observational studies in epidemiology (STROBE) statement: guidelines for reporting observational studies. Prev Med. 2007:45:247-51.

25. Boulet LP, FitzGerald JM, Levy ML, Cruz AA, Pedersen S, Haahtela T, Bateman ED. A guide to the translation of the Global Initiative for Asthma (GINA) strategy into improved care. Eur Respir J. 2012;39:1220-9.

26. Collage of Pulmonologists. Guideline of the Ministry of Health on the diagnosis, therapy and chronic care of bronchial asthma. Egészségügyi Közlöny. 2011;61:275.

27. WHO. WHO collaborating centre for drug statistics methodology. ATC/ DDD index. Geneva: WHO; 2016.

28. Topalovic M, Derom E, Osadnik CR, Troosters T, Decramer M, Janssens W. Airways resistance and specific conductance for the diagnosis of obstructive airways diseases. Respir Res. 2015;16:1.

29. Meguro M, Barley EA, Spencer S, Jones PW. Development and validation of an improved, COPD-specific version of the St. George Respiratory questionnaire. CHEST J. 2007;132:456-63.

30. Miller MR, Hankinson J, Brusasco V, Burgos F, Casaburi R, Coates A, Crapo R, Enright P, van der Grinten CP, Gustafsson P, Jensen R, Johnson DC, MacIntyre N, McKay R, Navajas D, Pedersen OF, Pellegrino R, Viegi G, Wanger J. ATS/ERS task force. Standardisation of spirometry. Eur Respir J. 2005;26:319-38

31. Wanger J, Clausen J, Coates A, Pedersen O, Brusasco V, Burgos F, Casaburi R, Crapo R, Enright P, Van Der Grinten C. Standardisation of the measurement of lung volumes. Eur Respir J. 2005;26:511.

32. Stocks J, Godfrey S, Beardsmore C, Bar-Yishay E, Castile R. ERS/ATS task force on standards for infant respiratory function testing. european respiratory society/American Thoracic Society. Plethysmographic measurements of lung volume and airway resistance. ERS/ATS task force on standards for infant respiratory function testing. European Respiratory Society/American Thoracic Society. Eur Respir J. 2001;17:302-12.

33. Jones PW, Quirk F, Baveystock C. The St George's respiratory questionnaire. Respir Med. 1991;85:25-31.

34. Jones PW. St. George's respiratory questionnaire: MCID. COPD J Chronic Obstructive Pulmonary Disease. 2005;2:75-9.

35. Quanjer PH. Standardized lung function testing: report working party" Standardization of Lung Function Tests", European Community for coal and steel. Luxembourg: Pergamon press; 1983.
36. Contoli M, Bousquet J, Fabbri L, Magnussen H, Rabe K, Siafakas N, Hamid Q, Kraft M. The small airways and distal lung compartment in asthma and COPD: a time for reappraisal. Allergy. 2010;65:141-51.

37. Contoli M, Santus P, Papi A. Small airway disease in asthma: pathophysiological and diagnostic considerations. Curr Opin Pulm Med. 2015;21:68-73.

38. Wells SM, Holian A. Asymmetric dimethylarginine induces oxidative and nitrosative stress in murine lung epithelial cells. Am J Respir Cell Mol Biol. 2007:36:520-8.

39. Ueda S, Kato S, Matsuoka H, Kimoto M, Okuda S, Morimatsu M, Imaizumi T. Regulation of cytokine-induced nitric oxide synthesis by asymmetric dimethylarginine: role of dimethylarginine dimethylaminohydrolase. Circ Res. 2003;92:226-33.

40. Tsikas D, Sandmann J, Savva A, Lueßen P, Böger RH, Gutzki F, Mayer B, Frölich JC. Assessment of nitric oxide synthase activity in vitro and in vivo by gas chromatography-mass spectrometry. J Chromatogr B Biomed Sci Appl. 2000;742:143-53.

41. Carraro S, Giordano G, Piacentini G, Kantar A, Moser S, Cesca L, Berardi M, Di Gangi IM, Baraldi E. Asymmetric dimethylarginine in exhaled breath condensate and serum of children with asthma. CHEST J. 2013;144:405-10

42. Lau EM, Morgan PE, Belousova EG, Toelle BG, Ayer JG, Celermajer DS, Marks GB. Asymmetric dimethylarginine and asthma: results from the Childhood Asthma Prevention Study. Eur Respir J. 2013;41:1234-7.

43. Usmani OS. Small-airway disease in asthma: pharmacological considerations. Curr Opin Pulm Med. 2015;21:55-67.

44. Baraldo S, Saetta M, Cosio MG. Pathophysiology of the small airways. Semin Respir Critical Care Med. 2003;24:465-72.

45. Yanai M, Sekizawa K, Ohrui T, Sasaki H, Takishima T. Site of airway obstruction in pulmonary disease: direct measurement of intrabronchial pressure. J Appl Physiol. 1985;1992(72):1016-23.

46. Barley E, Jones P. A comparison of global questions versus health status questionnaires as measures of the severity and impact of asthma. Eur Respir J. 1999;14:591-6.

47. Koch B, Friedrich N, Voelzke H, Joerres RA, Felix SB, Ewert R, Schaeper C, Glaeser S. Static lung volumes and airway resistance reference values in healthy adults. Respirology. 2013;18:170-8.

\section{Submit your next manuscript to BioMed Central and we will help you at every step:}

- We accept pre-submission inquiries

- Our selector tool helps you to find the most relevant journal

- We provide round the clock customer support

- Convenient online submission

- Thorough peer review

- Inclusion in PubMed and all major indexing services

- Maximum visibility for your research

Submit your manuscript at www.biomedcentral.com/submit
() Biomed Central 